\title{
ORIGINAL
}

\section{ZOONOSIS EMERGENTES LIGADAS A ANIMALES DE COMPAÑÍA EN LA COMUNIDAD DE MADRID: DISEÑO DE UN MÉTODO PARA ESTABLECER PRIORIDADES EN SALUD PÚBLICA}

\author{
Almudena García Nieto (1), Guillermo Medina Blanco (2) y Javier Reinares Ortiz de Villajos (3) \\ (1) Servicio de Sanidad Ambiental. Instituto de Salud Pública. Comunidad de Madrid \\ (2) Centro de Salud Pública de Alcobendas. Instituto de Salud Pública. Comunidad de Madrid \\ (3) Centro de Salud Pública de Alcalá de Henares. Instituto de Salud Pública. Comunidad de Madrid
}

\section{RESUMEN}

Fundamento: La emergencia y re-emergencia de enfermedades infecciosas revisten una gran preocupación en Salud Pública. Las peculiaridades socio-demográficas de la Comunidad de Madrid hacen que deba prestarse una mayor atención a las zoonosis transmitidas por animales de compañía. Los objetivos del trabajo fueron mejorar el conocimiento de las zoonosis emergentes y re-emergentes transmitidas por animales de compañía y el diseño de un método para la priorización de éstas.

Métodos: A partir de las enfermedades recogidas de fuentes oficiales, laboratorios y revisión bibliográfica se aplicó un método de cuantificación y ponderación diseñado por el grupo de trabajo y adaptado a la especificidad del estudio.

Resultados: Del análisis de 137 enfermedades, 24 cumplieron los criterios de admisión. El método de ponderación se refleja en una tabla que recoge once criterios de puntuación, sus categorías y lo coeficientes adoptados. La Salmonelosis fue la sus carmegoní. rar, seguida de Fiebre Q, Tularemia e Infección por Hantavirus.

Conclusiones: Se ha diseñado un método de evaluación específico de enfermedades emergentes y re-emergentes que permite establecer prioridades en el campo de la planificación en Salud Pública. El presente estudio aporta una relación, por orden de importancia, de 24 zoonosis sobre las que se deberán plantear nuevas líneas estratégicas para su investigación y/o control.

Palabras clave: Zoonosis. Enfermedades transmisibles emergentes. Método de prioridades. Animales domésticos.

Correspondencia:

Almudena García Nieto

Instituto de Salud Pública.

C/ Julián Camarillo 4. Edificio B. $2^{\text {a Planta. }}$

28037 Madrid.

Correo electrónico: almudena.garcia@madrid.org

\section{ABSTRACT}

Emerging Zoonoses Linked to Pets in the Autonomous Community of Madrid: Design of a Method for Setting Public Health Priorities. Spain

Background: The emergence and re-emergence of infectious diseases are a major concern in Public Health. The unique sociodemographic aspects of the Autonomous Community of Madrid make it necessary for a greater deal of attention to be paid to pettransmitted zoonoses. This study is aimed as heightening the knowledge of the emerging and re-emerging pet-transmitted zoonoses and the design of a method for prioritizing the same.

Methods: Based on the diseases obtained from official sources, laboratories and a review of published studies, a quantification and weighting method designed by the working group and adapted to the specificity of this study was applied.

Results: Based on the analysis of 137 diseases, 24 met the admission requirements. The weighting method is provided in table form, including eleven scoring criteria, the criteria categories and the coefficients employed. Salmonellosis was the top-ranked disease, followed by Q Fever, Tularemia and Hantavirus Infection.

Conclusions: A method for specifically evaluating emerging and re-emerging diseases was designed, affording the possibility of setting priorities in the Public Health planning field. This study provides a listing of 24 zoonoses ranked in order of importance, based on which new strategy-related lines must be set out for the research and/or control thereof. Pets.

Key words: Zoonoses. Communicable Diseases Emerging. 


\section{INTRODUCCIÓN}

Las enfermedades infecciosas emergentes se definen como aquéllas que han aparecido por vez primera en una población, o que existiendo en la misma están incrementando rápidamente su incidencia y rango geográfi$\mathrm{co}^{1}$. Las enfermedades infecciosas re-emergentes son aquéllas enfermedades conocidas que habían descendido a niveles muy bajos y que se incrementan o reaparecen ${ }^{1}$. Los términos zoonosis emergentes y re-emergentes son empleados para las enfermedades que se transmiten de forma natural entre los animales vertebrados y el hombre.

Existen numerosas razones que explican la aparición de zoonosis emergentes y la reemergencia de otras que se creían ya controladas: la resistencia a los tratamientos; el aumento del tráfico de viajeros y mercancías; los cambios en los sistemas de producción animal; los cambios tecnológicos en el comercio de alimentos, en la manipulación o en su procesamiento; las alteraciones del medio ambiente que producen desequilibrios y la creación de nuevos «nichos» ecológicos (la intrusión del hombre en medios selváticos por motivos de ocio o trabajo, la deforestación, la introducción de especies exóticas, etc.); el aumento de la población inmunodeprimida; los cambios en hábitos sociales; el incremento de espacios de uso colectivo que favorecen la difusión de algunas enfermedades (asilos, guarderías, la vida en grandes ciudades, etc.); la aparición de nuevos agentes infecciosos o la mutación de otros conocidos; la reorientación de las medidas de Salud Pública en detrimento de las enfermedades infecciosas, etc. ${ }^{2}$.

En la Asamblea Mundial de la Salud de 1995, los estados miembros de la Organización Mundial de la Salud (OMS) expresaron la preocupación existente ante este problema e instaron a que se reforzara la vigilancia de las enfermedades infecciosas así como a que se obtuviera información acerca de las mismas. Con estos fines fue creada la División de Vigilancia y Control de Emergentes y Otras Enfermedades Contagiosas ${ }^{3}$.

Según los datos del Padrón Municipal de habitantes de 2001 la Comunidad de Madrid cuenta con una población de 5.372.433 habitantes concentrada fundamentalmente en el municipio de Madrid y otros de la corona metropolitana, residiendo solamente un $7,99 \%$ en municipios con población inferior a 20.000 habitantes ${ }^{4}$, lo que denota el marcado carácter urbano de su población. Su superficie de aproximadamente $8.000 \mathrm{~km}^{2}$ da lugar a una densidad de población de 669 habitantes por kilómetro cuadrado, la más elevada del resto de comunidades del estado español.

La encuesta del Sistema de Vigilancia de Factores de Riesgo asociados a Enfermedades No Transmisibles dirigido a población juvenil de la Comunidad de Madrid ${ }^{5}$, cifró que en el $52,9 \%$ de los hogares en los que viven jóvenes se convive con animales de compañía ${ }^{6}$, el 36,2\% de una sola especie, el $11,8 \%$ de dos y el $5 \%$ de tres o más ${ }^{7}$.

Estas particularidades socio-demográficas del área de estudio hacen de sumo interés epidemiológico las zoonosis ligadas a animales de compañía.

La Comisión Técnica de los Programas de Vigilancia y Control de Antropozoonosis de la Consejería de Sanidad de la Comunidad de Madrid creó en 1998 un grupo de trabajo sobre las zoonosis emergentes y re-emergentes ligadas a animales de compañía, cuya vía de transmisión no fuera exclusivamente alimentaria, con el propósito de iniciar su estudio y valoración.

Los objetivos del presente trabajo son: el conocimiento de las zoonosis ligadas a los animales de compañía, el establecimiento de un método de cuantificación y ponderación de zoonosis emergentes y re-emergentes y la priorización de éstas en la Comunidad de Madrid. 


\section{MATERIAL Y MÉTODOS}

\section{Recogida y análisis de información}

Se emplearon como fuentes de información los sistemas oficiales que integran la red regional y nacional de vigilancia epidemiológica $^{8,9,10}$ : Enfermedades de Declaración Obligatoria (EDO), Notificación de Alertas y Brotes Epidémicos y Sistema de Información Microbiológica (SIM). Además contribuyeron voluntariamente 8 clínicas veterinarias (3 ubicadas en el municipio de Madrid y el resto en municipios de la corona metropolitana) y 3 laboratorios oficiales de sanidad animal, que aportaron información relativa a las patologías más frecuentemente diagnosticadas. Paralelamente se inició un proceso de búsqueda bibliográfica. En total se revisaron más de 200 referencias para la elaboración del presente artículo.

Para la selección de los agentes contemplados en las fuentes de información se establecieron los siguientes criterios de exclusión:

a) Agentes no zoonóticos y/o agentes en los que no había datos concluyentes sobre su posible carácter zoonótico: Gastrospirilum hominis, Helicobacter $s p$, Virus de la Leucemia Felina, etc.

b) Agentes de transmisión exclusivamente alimentaria: Bacillus cereus, Listeria sp, etc.

c) Agentes con programas específicos de control o actividades concretas dentro de éstos, llevados a cabo por la Consejería de Sanidad de la Comunidad de Madrid: leishmaniosis, hidatidosis, brucelosis, rabia, triquinelosis y tuberculosis.

A partir de la relación de enfermedades contempladas en las fuentes de información de referencia se inició su estudio basado en la consulta y revisión de diversa documentación científica. Además, la documentación bibliográfica permitió la incorporación de otros agentes patógenos que fueron considerados de interés por el grupo en función de un conjunto de resultados objetivos, los cuales hipotéticamente planteaban la posibilidad de su presencia y/o presentación en nuestro entorno (morbilidad, condiciones ambientales óptimas, presencia de reservorios y vectores, distribución geográfica de casos próximos a nuestra comunidad, etc.).

En está primera etapa fueron documentadas y analizadas 137 enfermedades, siendo finalmente 24 las seleccionadas, con sus correspondientes fichas informativas.

\section{Diseño de un modelo de priorización de enfermedades: criterios de aplicación}

Se adoptó como método de referencia el modelo empleado en Canadá en 1991 para el establecimiento de prioridades en los programas de control de enfermedades infecciosas $^{11}$, el cual permite determinar la importancia de una enfermedad mediante la cuantificación y ponderación de 12 criterios preestablecidos por un grupo de expertos. A partir de este método y en consonancia con las particularidades del presente estudio, se realizó una reorientación del mismo, siendo seleccionados los siguientes criterios: letalidad, transmisibilidad, capacidad de producción de brotes, importancia socio-económica, percepción social del riesgo, posibilidad de prevención por vacunación y necesidad de medidas urgentes de Salud Pública.

Fueron desestimados dos de los criterios: importancia dada a la enfermedad por la Administración Agrícola de Canadá, y mortalidad. En el primer caso, por su escasa contribución al objetivo del estudio. En el segundo, por las limitaciones en la obtención de datos fiables, dado que, la mayoría de las enfermedades estudiadas son raras en nuestro entorno y su medida de frecuencia en las dife- 
rentes fuentes consultadas (frecuencias absolutas, población de referencia indeterminada, etc.) era imprecisa y heterogénea, imposibilitando su cuantificación y comparación. No obstante, se incluía un criterio relacionado con la mortalidad: la letalidad, mucho más preciso ya que mediante este indicador se estima el número de muertes en relación al conjunto de enfermos, mientras que en el de mortalidad, el número de muertes se relaciona con el conjunto de individuos que integran la población a la que pertenecen.

Además, se optó por otro tipo de enfoque en tres de los criterios: morbilidad, importancia dada por la OMS e incidencia. La morbilidad fue sustituida por diagnósticos de laboratorio y estimada a partir de la detección de los agentes patógenos mediante aislamientos y/o por encuestas serológicas, debido nuevamente a la dificultad de obtener datos fiables y precisos (no se concretaba si se trataba de casos sospechosos, probables o confirmados, ni la fuente a partir de la cual era estimada: número de días de hospitalización, diagnósticos al alta hospitalaria, etc.). Respecto a la importancia dada por la OMS se sustituyó por la importancia dada por la Administración Sanitaria de la Comunidad de Madrid. En cuanto a la incidencia se incluyó la valoración de la tendencia evolutiva de la enfermedad en nuestro entorno en los diez últimos años como ya se hiciera en Francia (1994 y 1995) ${ }^{12}$.

Por último, se vio recomendable incluir un criterio que también era contemplado en el trabajo francés: capacidad de intervención preventiva y clínica.

En definitiva se adoptaron once criterios de valoración, destacando algunas precisiones sobre los siguientes:

1. Incidencia: Expresada a través de la tasa de incidencia (número de casos nuevos notificados que se producen en un periodo determinado referida a la población diana), considerando caso aquel que presenta sintomatología clínica y confirmación de laboratorio.

2. Letalidad: Expresada a través de la tasa de letalidad en países desarrollados (número de muertes en relación con la población de enfermos).

3. Capacidad de producción de brotes: Agrupación de dos o más casos en el tiempo y en el espacio con un origen de infección común. No se contemplaron aquéllos cuya vía de transmisión era a través de los alimentos y/o agua.

4. Percepción social del riesgo: Importancia que da la población a una enfermedad. Criterio subjetivo, basado en experiencias relacionadas con las manifestaciones de la población ante diferentes hechos relacionados con una enfermedad: alarma social, interés por tratamientos o vacunaciones, etc.

5. Importancia socio-económica: Costes de la enfermedad una vez instaurada: secuelas, incapacidad, hospitalización, tratamientos, bajas laborales, duración de la enfermedad, costes intangibles, etc. Dado que no existen estudios específicos en la mayoría de los casos estudiados, este criterio fue cuantificado bajo una interpretación subjetiva del grupo de trabajo, aunque fundamentada en la bibliografía consultada.

6. Necesidad de medidas inmediatas de Salud Pública: Intervención urgente para evitar la transmisibilidad y reducir la alarma social.

Establecidos y definidos los once criterios con los que se puntuarían las enfermedades, y con el fin de evitar la valoración de los agentes de modo subjetivo, se establecieron categorías acordes a las definiciones de cada criterio, siéndoles aplicados coeficientes de ponderación según la importancia que habían recibido. Además, con el fin de normalizar 
algunos criterios se establecieron coeficientes de corrección.

\section{Descripción y desarrollo del proceso de puntuación}

Con objeto de dotar de mayor operatividad a las fichas informativas de las enfermedades y facilitar su comparación y puntuación, se elaboraron otros nuevos adaptados a los 11 criterios reseñados. Estas fichas se actualizaron a medida que avanzaba el trabajo, de acuerdo con la bibliografía o documentación que se venía recogiendo.

Cada miembro del grupo analizó y puntuó de forma individualizada cada una de las enfermedades. En el caso de que existieran divergencias notables, se iniciaba un proceso de discusión y análisis, no anotando el resultado final hasta que no se llegaba a un consenso.

La calificación de las diferentes enfermedades se realizó criterio por criterio, es decir; en primer lugar se puntuó la importancia dada por la administración de cada uno de los agentes y a continuación se pasaba a la incidencia, y así sucesivamente, de este modo se pretendía evitar valorar de forma sesgada las enfermedades en función de la dinámica del grupo o de unas reuniones a otras.

Una vez concluido el proceso de puntuación se apreció que las enfermedades de transmisión fundamentalmente alimentaria y/o hídrica se encontraban sobrepuntuadas, con el fin de evitar esta distorsión en los resultados se aplicó un factor de corrección relacionado con esta vía de transmisión en el criterio relativo a la capacidad de transmisión del patógeno y se excluyó la misma del criterio de capacidad de producir brotes.

\section{RESULTADOS}

De la etapa de recogida y análisis de información se obtuvo un listado de 24 zoonosis: ancylostomiosis, babesiosis, campilobacteriosis, carbunco, criptosporidiosis, ehrlichiosis, enfermedad de Lyme, enfermedad de Newcastle, enfermedades transmitidas por mordedura (Bartonella sp., Spirillum minus, Streptobacillus moniliformes, Capnocytophaga sp., Pasteurella sp. y Virus B), fiebre exantemática del Mediterráneo, fiebre del Nilo Occidental, fiebre Q, fiebre recurrente transmitida por garrapatas, giardiosis, infección por hantavirus, leptospirosis, psitacosis, salmonelosis, sarna zoonótica, tiña zoonótica, toxocariosis, toxoplasmosis, tularemia y yersiniosis.

En la tabla 1 se presentan los criterios adoptados en el método de priorización con sus correspondientes categorías y los coeficientes de ponderación y de corrección establecidos. En la primera columna figuran los once criterios seleccionados y descritos anteriormente. En la segunda, están las características cualitativas o cuantitativas de cada criterio, denominadas categorías. La tercera columna incluye los valores numéricos otorgados a cada categoría y denominados coeficientes de ponderación, lógicamente cada criterio tiene un valor diferente según su importancia sanitaria: cinco de ellos (incidencia, letalidad, capacidad de transmisión del patógeno, capacidad de producir brotes y capacidad de intervención preventiva y clínica) tienen un máximo de 5 puntos, y otros criterios que se abren más a la subjetividad alcanzan un máximo de 2 puntos (importancia dada por la administración sanitaria, diagnósticos de laboratorio percepción social del riesgo, importancia socio-económica, necesidad de medidas inmediatas de salud pública). Por último, los coeficientes de corrección que se reflejan en la cuarta columna son valores numéricos que incluidos en algunos criterios deben de aplicarse sobre el coeficiente de ponderación.

La tabla 2 refleja la relación de las zoonosis prioritarias tras la aplicación de la metodología anteriormente descrita, la puntuación obtenida por cada una de ellas y aque- 
Tabla 1

Modelo de determinación y valoración de las zoonosis prioritarias en salud pública

\begin{tabular}{|c|c|c|c|c|}
\hline Criterios & \multicolumn{2}{|c|}{ Categorías del criterio } & $\begin{array}{l}\text { Coeficiente de } \\
\text { ponderación }\end{array}$ & Coeficientes de corrección \\
\hline $\begin{array}{l}\text { 1.- Importancia } \\
\text { dada por la } \\
\text { Administración } \\
\text { Sanitaria } \\
\text { (Valor máximo } \\
\text { 1 punto) }\end{array}$ & \multicolumn{2}{|c|}{$\begin{array}{l}\text { Existencia de actividades especificas, registros, etc. } \\
\text { Existencia de investigación, laboratorio de referencia }\end{array}$} & $\begin{array}{l}0-0,5 \\
0-0,5\end{array}$ & $\begin{array}{l}\text { Se puntúa en primer lugar con los datos existentes de la } \\
\text { Comunidad de Madrid, en ausencia de estos se puntúa con los } \\
\text { de España empleando un factor de corrección de } \pm \mathbf{0}, \mathbf{1} \text {. }\end{array}$ \\
\hline 2.- Incidencia & \multicolumn{2}{|c|}{$\begin{array}{l}\text { Muy Alta }(>1 / 100.000) \\
\text { Alta }(1 / 100.000-1 / 500.000) \\
\text { Media }(<1 / 500.000-1 / 1.000 .000) \\
\text { Baja }(<1 / 1.000 .000)\end{array}$} & $\begin{array}{c}5 \\
3 \\
2 \\
1 \\
\\
0,1-0,3 \\
0\end{array}$ & $\begin{array}{l}\text { Se puntúa en primer lugar con los datos existentes de la } \\
\text { Comunidad de Madrid; en ausencia de estos se puntúa con los } \\
\text { de España empleando un factor de corrección de } \pm \mathbf{0 , 2} \text {. A falta } \\
\text { de la información anterior, se establece que la incidencia está } \\
\text { Sin Determinar o bien es Desconocida empleando un factor } \\
\text { de corrección de } \mathbf{0 , 5} \text { cuando se recurra a datos de Países } \\
\text { Mediterráneos, de } \mathbf{0 , 1} \text { cuando sea de otros Países Europeos y } \\
\text { sin coeficiente de corrección si se utilizara de otras áreas } \\
\text { geográficas distintas a éstas. } \\
\text { Tendencia de la incidencia en los últimos diez años } \pm \mathbf{1} \text {. }\end{array}$ \\
\hline $\begin{array}{l}\text { 3.- Diagnósticos } \\
\text { de laboratorio } \\
\text { (Valor máximo } \\
\text { 1 punto) }\end{array}$ & $\begin{array}{l}\text { Aislamientos } \\
\text { Seroprevalencia } \\
\text { Desconocida }\end{array}$ & $\begin{array}{l}\geq 50 / \text { año } \\
<50 / \text { año } \\
\geq 25 \% \\
10-25 \% \\
\leq 10 \%\end{array}$ & $\begin{array}{c}1 \\
0,5 \\
1 \\
0,75 \\
0,5 \\
0\end{array}$ & $\begin{array}{l}\text { Se puntúa en primer lugar con los datos existentes de la } \\
\text { Comunidad de Madrid; en ausencia de estos se puntúa con los } \\
\text { de España empleando un factor de corrección de } \pm \mathbf{0 , 2} \text {. A falta } \\
\text { de la información anterior, se establece que la información de } \\
\text { Diagnósticos Laboratoriales es Desconocida empleando un } \\
\text { factor de correción de } \mathbf{0}, \mathbf{5} \text { cuando se recurra a datos de Páses } \\
\text { Mediterráneos, de } \mathbf{0 , 1} \text { cuando sea de otros Países Europeos y } \\
\text { sin coeficiente de corrección si se utilizara de otras áreas } \\
\text { geográficas distintas a éstas. }\end{array}$ \\
\hline 4.- Letalidad & $\begin{array}{l}\text { Muy Alta } \\
\text { Alta } \\
\text { Baja } \\
\text { Muy Baja } \\
\text { Rara } \\
\text { Nula }\end{array}$ & $\begin{array}{l}>20 \% \\
20-5,01 \% \\
5-2,01 \% \\
2-1 \% \\
<1 \%\end{array}$ & $\begin{array}{l}5 \\
4 \\
3 \\
2 \\
1 \\
0\end{array}$ & \\
\hline $\begin{array}{l}\text { 5.- Capacidad de } \\
\text { Transmisión } \\
\text { del patógeno } \\
\text { (Valor máximo } \\
5 \text { puntos) }\end{array}$ & $\begin{array}{c}\text { Vías de Contagio: } \\
\text {-Directas } \\
\text {-Indirectas }\end{array}$ & $\begin{array}{l}\text { Urbano (doméstico, compañía) } \\
\text { Salvaje } \\
\text { Inanimado (suelo, etc.) } \\
\text { - Aerógena } \\
\text { - Digestiva } \\
\text { - Cutánea } \\
\text { - Vectores (garrapatas, etc.) } \\
\text { - Mecánica (fómites, moscas, } \\
\text { etc ) }\end{array}$ & $\begin{array}{r}0,5 \\
0,25 \\
0,25 \\
\\
0,5 \\
0,5 \\
0,5 \\
0,5 \\
0,5\end{array}$ & $\begin{array}{l}\text { En las vías por contagio directo, el sumatorio ha de ser como } \\
\text { máximo de } 1 \text { punto } \\
\text { Papel principal de los alimentos en la transmisión: -1 }\end{array}$ \\
\hline & $\begin{array}{l}\text { Contagio Interhun } \\
\text {-Directo- Fecal-or } \\
\text { trasplac } \\
\text {-Indirecto- Transfi }\end{array}$ & $\begin{array}{l}\text { lano: } \\
\text { l, aerógeno, saliva, transplante, vectores, etc }\end{array}$ & $\begin{array}{l}1 \\
1\end{array}$ & \\
\hline $\begin{array}{l}\text { 6.- Capacidad de } \\
\text { Producción de } \\
\text { Brotes }\end{array}$ & $\begin{array}{l}\text { Muy Alta } \\
\text { Alta } \\
\text { Baja } \\
\text { Alta Profesional } \\
\text { Muy Baja } \\
\text { Esporádica } \\
\text { Esporádica Profesio }\end{array}$ & & $\begin{array}{c}5 \\
4 \\
3 \\
2,5 \\
2 \\
1 \\
0,5\end{array}$ & $\begin{array}{l}\text { Otros grupos de riesgo no ocupacionales: actividades } \\
\text { recreativas, de ocio, etc. } \mathbf{- 0 , 5}\end{array}$ \\
\hline $\begin{array}{l}\text { 7.- Percepción } \\
\text { social del } \\
\text { riesgo }\end{array}$ & $\begin{array}{l}\text { Alta } \\
\text { Baja } \\
\text { Nula }\end{array}$ & & $\begin{array}{c}1,5 \\
0,75 \\
0\end{array}$ & \\
\hline $\begin{array}{l}\text { 8.- Importancia } \\
\text { Socio- } \\
\text { económica }\end{array}$ & $\begin{array}{l}\text { Muy Alta } \\
\text { Alta } \\
\text { Media } \\
\text { Baja } \\
\text { Muy Baja }\end{array}$ & & $\begin{array}{c}2 \\
1,5 \\
1 \\
0,75 \\
0,5\end{array}$ & \\
\hline $\begin{array}{l}\text { 9.- Posibilidad de } \\
\text { Prevención } \\
\text { por vacuna }\end{array}$ & $\begin{array}{l}\text { Vacuna efectiva sol } \\
\text { Vacuna poco efecti } \\
\text { No Existe vacuna }\end{array}$ & $\begin{array}{l}\text { o Población de Riesgo } \\
\text { va y no específica }\end{array}$ & $\begin{array}{l}2 \\
1 \\
0\end{array}$ & \\
\hline $\begin{array}{l}\text { 10.- Capacidad } \\
\text { de } \\
\text { Intervención } \\
\text { Preventiva y } \\
\text { Clínica }\end{array}$ & $\begin{array}{l}\text { Clinica Nula/Prever } \\
\text { Clinica Baja/Prever } \\
\text { Clínica Alta/Preven } \\
\text { Clinica Nula/Prever } \\
\text { Clinica Baja/Prever } \\
\text { Clínica Alta/Preven } \\
\text { Clinica Nula/Prever } \\
\text { Clinica Baja/Prever } \\
\text { Clínica Alta/Preven }\end{array}$ & $\begin{array}{l}\text { tiva Alta } \\
\text { tiva Alta } \\
\text { tiva Alta } \\
\text { tiva Baja } \\
\text { tiva Baja } \\
\text { tiva Baja } \\
\text { tiva Nula } \\
\text { tiva Nula } \\
\text { tiva Nula }\end{array}$ & $\begin{array}{c}5 \\
4,5 \\
4 \\
3 \\
2,5 \\
1,5 \\
1.5 \\
1 \\
0\end{array}$ & \\
\hline $\begin{array}{l}\text { 11.- Necesidad de } \\
\text { Medidas } \\
\text { Inmediatas de } \\
\text { Salud Pública } \\
\end{array}$ & $\begin{array}{l}\mathrm{Si} \\
\text { No }\end{array}$ & & $\begin{array}{l}1 \\
0\end{array}$ & \\
\hline
\end{tabular}


Tabla 2

Zoonosis ligadas a los animales de compañía prioritarias en la Comunidad de Madrid

\begin{tabular}{|c|c|c|}
\hline Enfermedad & Puntuación & Criterios relevantes \\
\hline Salmonelosis & 19,5 & $\begin{array}{l}\text { - Incidencia muy alta } \\
\text { - Letalidad del } 7 \% \text { pediátrica y del } 8,7 \% \text { geriátrica } \\
\text { - Alta capacidad de transmisión } \\
\text { - Elevada percepción social del riesgo e importancia socio-económica }\end{array}$ \\
\hline Fiebre Q & 17,5 & $\begin{array}{l}\text { - Incidencia alta } \\
\text { - Vacuna eficaz en población de riesgo }\end{array}$ \\
\hline Tularemia & 17,35 & $\begin{array}{l}\text { - Alta importancia dada por la administración } \\
\text { - Incidencia alta } \\
\text { - Capacidad de producir brotes } \\
\text { - Posibilidad de prevención por vacuna }\end{array}$ \\
\hline Infección por Hantavirus & 16,15 & $\begin{array}{l}\text { - Letalidad alta } \\
\text { - Capacidad de producir brotes }\end{array}$ \\
\hline Carbunco & 15,7 & $\begin{array}{l}\text { - Alta capacidad de transmisión } \\
\text { - Posibilidad de prevención por vacuna } \\
\text { - Alta capacidad de intervención preventiva y clínica }\end{array}$ \\
\hline Psitacosis & 15,5 & $\begin{array}{l}\text { - Enfermedad ocupacional } \\
\text { - Capacidad de producción de brotes }\end{array}$ \\
\hline Toxoplasmosis & 15,05 & $\begin{array}{l}\text { - Alta capacidad de transmisión } \\
\text { - Importancia socio-económica relevante } \\
\text { - Alta capacidad de intervención preventiva }\end{array}$ \\
\hline Criptosporidiosis & 13,3 & $\begin{array}{l}\text { - Incidencia muy alta } \\
\text { - Letalidad muy baja o rara (excepto personas inmunodeprimidas) } \\
\text { - Capacidad de intervención clínica alta }\end{array}$ \\
\hline Leptospirosis & 12,95 & $\begin{array}{l}\text { - Incidencia baja } \\
\text { - Capacidad de producir brotes esporádicos } \\
\text { - Vacuna efectiva } \\
\text { - Baja capacidad de intervención preventiva }\end{array}$ \\
\hline Tiña zoonótica & 12,4 & $\begin{array}{l}\text { - Incidencia muy alta } \\
\text { - Alta capacidad de transmisión } \\
\text { - Alta capacidad de intervención clínica }\end{array}$ \\
\hline Campilobacteriosis & 12 & $\begin{array}{l}\text { - Incidencia alta-muy alta } \\
\text { - Producción de brotes esporádicos } \\
\text { - Capacidad de intervención clínica alta }\end{array}$ \\
\hline Fiebre del Nilo Occidental & 11 & $\begin{array}{l}\text { - Incidencia sin determinar } \\
\text { - Letalidad alta } \\
\text { - Producción de brotes esporádicos } \\
\text { - Baja capacidad de intervención preventiva y clínica }\end{array}$ \\
\hline Fiebre exantemática del & 10,9 & - Incidencia alta \\
\hline Mediterráneo & & $\begin{array}{l}\text { - Brotes esporádicos ocupacionales } \\
\text { - Capacidad de intervención preventiva baja }\end{array}$ \\
\hline Sarna zoonótica & 10,85 & $\begin{array}{l}\text { - Incidencia alta-muy alta } \\
\text { - Alta capacidad de transmisión }\end{array}$ \\
\hline Enfermedad de Lyme & 10,75 & $\begin{array}{l}\text { - Incidencia baja } \\
\text { - Capacidad de prevención por vacuna }\end{array}$ \\
\hline $\begin{array}{l}\text { Fiebre Recurrente transmitida por } \\
\text { Garrapatas }\end{array}$ & 10,65 & $\begin{array}{l}\text { - Incidencia media } \\
\text { - Letalidad baja } \\
\text { - Brotes esporádicos ocupacionales }\end{array}$ \\
\hline Ehrlichiosis & 10,35 & $\begin{array}{l}\text { - Incidencia baja/sin determinar } \\
\text { - Letalidad baja }\end{array}$ \\
\hline $\begin{array}{l}\text { Enfermedades transmitidas por } \\
\text { mordedura }\end{array}$ & 9,75 & $\begin{array}{l}\text { - Incidencia baja } \\
\text { - Letalidad alta en algunas de las incluidas: Virus B (herpético } 1 \\
\text { cercophitecine) }\end{array}$ \\
\hline Yersiniosis & 9,2 & $\begin{array}{l}\text { - Elevado número de aislamientos anuales en Comunidad de Madrid } \\
\text { - Letalidad muy baja }\end{array}$ \\
\hline Toxocariosis & 9 & $\begin{array}{l}\text { - Incidencia baja/sin determinar } \\
\text { - Letalidad rara o nula }\end{array}$ \\
\hline Giardiosis & 8,5 & $\begin{array}{l}\text { - Incidencia alta/media } \\
\text { - Letalidad nula }\end{array}$ \\
\hline Babesiosis & 7,75 & $\begin{array}{l}\text { - Incidencia baja/sin determinar } \\
\text { - Letalidad rara }\end{array}$ \\
\hline Ancylostomiosis & 7,45 & $\begin{array}{l}\text { - Incidencia baja/sin determinar } \\
\text { - Letalidad nula }\end{array}$ \\
\hline Enfermedad de Newcastle & 5,2 & $\begin{array}{l}\text { - Incidencia sin determinar } \\
\text { - Leve }\end{array}$ \\
\hline
\end{tabular}


llos criterios que influyeron con más peso específico en la puntuación final. La enfermedad que obtuvo la mayor puntuación fue la salmonelosis $(19,5)$, por el contrario, en ultimo lugar se situó la enfermedad de Newcastle $(5,2)$ como problema sanitario de menor importancia. Las zoonosis que figuran en los primeros lugares coinciden con puntuaciones elevadas en aquellos criterios considerados relevantes y cuyos coeficientes de ponderación podían alcanzar un valor máximo de cinco puntos.

\section{DISCUSIÓN}

Se ha abordado una revisión de las zoonosis emergentes como planteamiento novedoso en la Comunidad de Madrid, mediante un método de priorización de enfermedades que define de forma objetiva la magnitud e importancia de cada enfermedad. El modelo de priorización diseñado posee características metodológicas originales que tienen en cuenta las singularidades de las zoonosis estudiadas.

Las limitaciones encontradas en el desarrollo del trabajo fueron numerosas, destacando especialmente la dificultad de obtener información sobre la situación de muchas enfermedades en nuestro entorno y la escasa fiabilidad de algunos datos recogidos, especialmente los relativos a la morbi-mortalidad, en particular la tasa de incidencia de las enfermedades, así como la comparación objetiva de la diversidad de datos de laboratorio. Por todo ello, el grupo de trabajo es consciente de que se han podido producir sesgos en la adopción de decisiones, en la puntuación de los agentes y consecuentemente en los resultados obtenidos.

El modelo diseñado para la priorización de las enfermedades, a diferencia de los métodos canadiense y francés, establece categorías que gradúan cada criterio y coeficientes de corrección en algunos de ellos que ajustan la puntuación, lo que permite mejorar la objetividad del resultado final.

El listado de enfermedades obtenidas no difiere notablemente del trabajo publicado recientemente por el Institut de Veille Sanitaire de Francia sobre zoonosis no alimentarias $^{13}$, salvo en la importancia y prioridad que se da a determinadas enfermedades. En este estudio, no limitado a animales de compañía, se seleccionaron 37 enfermedades entre las cuales figuran 17 de las 24 recogidas en la tabla 1 de resultados. Además, hay que tener en cuenta que nuestro trabajo no incluyó aquellas zoonosis sujetas a programas específicos de intervención, algunas de las cuales figuran en el trabajo francés.

En nuestro caso el orden en el que aparecen las enfermedades no tiene por qué llevar aparejada una inmediata intervención en salud pública, ya que las líneas estratégicas a desarrollar podrán ser muy diferentes según cada enfermedad o grupos de enfermedades.

Referente a la relación de zoonosis resultantes, hay que tener en cuenta que algunas de ellas han estado vinculadas tradicionalmente a animales de abasto, como es el caso de salmonelosis, carbunco, fiebre Q, campilobacteriosis, etc.... Los nuevos hábitos y estilos de vida de una sociedad eminentemente urbana implican la necesidad de reflexionar y valorar el papel que los animales de compañía representan en su casuística, así como en los patrones epidemiológicos de estas enfermedades. En el caso concreto de la salmonelosis se deberá tener en cuenta a los reptiles y anfibios como fuentes de contagio para el hombre y por lo tanto incluir a éstos en la investigación epidemiológica ante la presentación de casos. En ese sentido otros países como Canadá o Estados Unidos, que han cuantificado la magnitud de las salmonelosis transmitidas por reptiles, han visto necesario adoptar regulaciones específicas que han permitido frenar y disminuir considerablemente la casuística, mediante medidas de información en establecimientos 
de venta o la prohibición de la presencia de estos animales en centros infantiles ${ }^{14}$.

En algunas de las zoonosis valoradas, como psitacosis y tularemia, los resultados obtenidos confirman una situación conocida por el grupo de trabajo, ya que la periódica presentación de casos ha implicado diferentes intervenciones desde los servicios de salud pública. En este sentido, se deberán diseñar protocolos de actuación con objeto de mejorar la investigación epidemiológica y adoptar las adecuadas medidas de prevención y control.

Respecto a ciertas enfermedades como: fiebre del Nilo, infección por hantavirus, ehrlichiosis o infección por virus B herpético (incluido dentro de las enfermedades transmitidas por mordedura), aunque en la actualidad aún no ha sido constatada su presencia en nuestro país, con alguna salvedad al respecto, la severidad de los cuadros clínicos con que se presentan, su elevada letalidad, la inexistencia de tratamientos eficaces, etc., ha suscitado una honda preocupación por parte de la comunidad científica, de tal modo que algunas de ellas están incluidas en la actualidad en proyectos de investigación y en redes temáticas de investigación cooperativa como la Red de enfermedades víricas transmitidas por artrópodos y roedores (EVITAR) o la Red de enfermedades bacterianas transmitidas por garrapatas (EBATRAG). Los propósitos del grupo de trabajo al respecto serán el apoyo y participación en los mismos. Algunos países, siguiendo las recomendaciones de la OMS, han puesto en marcha estrategias específicas frente a las enfermedades infecciosas emergentes, entre las que cabría destacar el modelo seguido en Alemania $^{15}$. En este sentido, nuestros resultados posibilitan diferentes vías de trabajo, con actuaciones diversas entre las que cabe destacar: la actualización de los sistemas de vigilancia, la promoción de la investigación en enfermedades cuya situación actual es desconocida pese a existir indicios de su presencia y la revisión de algunas zoonosis que aún formando parte de los programas de Salud Pública, quizá no se esté investigando suficientemente el papel epidemiológico que juegan los animales de compañía.

\section{AGRADECIMIENTOS}

Queremos agradecer a cuantos de una $\mathrm{u}$ otra forma han participado en el presente trabajo y de modo particular a la Dra. Rosario Castañeda, que colaboró durante un prolongado periodo aportando numerosas opiniones que enriquecieron el mismo. Además no podemos olvidar el interés mostrado en todo momento por la Jefatura de Sección de Zoonosis y Riesgos Biológicos por la marcha del equipo de trabajo.

\section{BIBLIOGRAFÍA}

1. Morse SS. Factors in the emergence of infectious diseases. Emerg Infect Dis 1995 Jan- Mar; 1(1):715. http://www.cdc.gov/ncidod/eid/index.htm

2. Word Health Organization. Emerging and Reemerging infectious diseases. Information Fact Sheet 97. Revised August 1998. http://www.who. int/inf-fs/en/fact097.html

3. Word Health Organization. Report of the Second WHO Meeting on Emerging Infectious Diseases. Ginebra: Word Health Organization; 1995.

4. Instituto de Estadística de la Comunidad de Madrid. Anuario Estadístico de la Comunidad de Madrid 2003. $1^{a}$ ed. Madrid: Consejería de Economía e Innovación Tecnológica; 2002.

5. Consejería de Sanidad y Servicios Sociales. Hábitos de salud en la población juvenil de la Comunidad de Madrid 1999. Boletín Epidemiológico de la Comunidad de Madrid 1999; 6(6): 3-31.

6. Boletín Oficial de la Comunidad de Madrid. Ley 1/1990, de 1 de febrero, de Protección de los Animales Domésticos. BOCM núm 39, 15/2/1990: 3.

7. Moreno Alcalde S, Galán Labaca I, Martínez Cortés M, Castañeda López R y García Nieto A. Convivencia con animales en la población juvenil de la Comunidad de Madrid. Profesión Veterinaria 2001; 49: $38-40$ 
8. Boletín Oficial del Estado. Real Decreto 2210/1995 por el que se crea la Red Nacional de Vigilancia Epidemiológica. BOE núm 21, 24/1/1996

9. Boletín Oficial de la Comunidad de Madrid. Decreto $184 / 1996$ por el que se crea la Red de Vigilancia Epidemiológica de la Comunidad de Madrid. BOCM núm 2, 3/1/1997.

10. Boletín Oficial de la Comunidad de Madrid. Orden 9/1997 para el desarrollo del Decreto 184/1996, en lo que se refiere a las Enfermedades de Declaración obligatoria, a las situaciones Epidémicas y Brotes, y al Síndrome de Inmunodeficiencia Adquirida (SIDA) e Infección por virus de la Inmunodeficiencia Humana (VIH). BOCM núm 18, 22/1/1997.

11. Health and Welfare Canada. Establishing goals, techniques and priorities for national communicable disease surveillance. Can Dis Wkly Rep 1991 Apr 20; 17(16): 79-84.
12. Hubert B, Haury B. Orientations pour la révision des modalités de surveillance des maladies transmissibles en France. Bolletin epidémiologique hebdomadaire 1996; 26:115-117.

13. Définition des priorités dans le domaine des zoonoses non alimentaires 2000-2001. Institut de Veille Sanitaire. www.invs.sante.fr/publications/2002/ def_priorite_zoonoses/index.html

14. CDC. Reptile-Associated salmonellosis-Selected States, 1998-2002. Morb Mortal Wkly Rep 2003; 52(49):1206-1209. www.cdc.gov/mmwr/preview/mmwrhtml/mm5249a3.htm

15. Developing National Epidemiological Capacity to Meet the Challenges of Emerging Infections in Germany. Petersen LR, Ammon A, Hamouda O, Breuer T, Kießling S, Bellach B et al. Emerg Infect Dis 2000 Nov-Dec; 6(6): 576-584. http://www.cdc. gov/ncidod/eid/vol6no6/petersonG2.ht 